\title{
Unusual Sensory Disturbances Revealing Posterior Spinal Artery Infarct
}

\author{
Fabrice Vuillier ${ }^{a, b}$ Laurent Tatu $^{a, b} \quad$ Ansoumane Camara ${ }^{a}$ \\ Emeline Muzard ${ }^{a}$ Thierry Moulin ${ }^{a}$
}

${ }^{a}$ Service de Neurologie 2, Centre Hospitalier Universitaire de Besançon, et

${ }^{b}$ Département d'Anatomie, UFR Sciences Médicales et Pharmaceutiques,

Université de Franche-Comté, Besançon, France

\section{Key Words}

Spinal cord infarct · Posterior spinal artery - Sensory stroke $\cdot$ Magnetic resonance imaging $\cdot$ Clinical correlations

\begin{abstract}
Spinal cord infarctions are rare, especially at the cervical level and in the posterior spinal artery territory. Moreover, this diagnosis is difficult to establish as the clinical picture varies. Even if sensory patterns appear as cardinal signs, their distribution can be very variable. We report a rare case of posterior cervical cord infarction revealed by an unusual pure sensory clinical picture.
\end{abstract}

\section{Introduction}

Spinal cord infarctions are rare, especially at the cervical level [1, 2]. Posterior spinal infarctions are less common than those located in the anterior territory, due to a pial collateral network and the dual posterior spinal artery [2]. In most cases, sensory patterns are present, but their modalities of expression and systematisation vary. We report a rare case of posterior cervical cord infarction revealed by an unusual pure sensory clinical picture.

\section{Case Report}

The patient was a 41-year-old male with a history of dyslipidaemia and arterial hypertension for which he had received treatment. Five days after a motorcycle accident, he suddenly developed acute paraesthesia in the left occipital and temporal area of the head and the left side of the nape of the neck. A few hours later, the paraesthesia extended towards the left side of the face. These symptoms 
persisted and, subsequently, sensory changes spread over the entire left side of the trunk and the left arm. He was hospitalised $8 \mathrm{~h}$ after the onset of symptoms.

At the time of admission, the sensory symptoms persisted and the physical examination was normal. All sensory modalities, such as pinprick, touch, vibratory sense and position sense, were normal, and no motor abnormalities were found in the head, neck, trunk and limbs. The patient did not have any pain, dysphagia, vertigo or dizziness, nausea or vomiting, Horner's syndrome, gait ataxia or urinary retention (National Institutes of Health Stroke Scale $=0$ ). Arterial blood pressure was $170 / 90 \mathrm{~mm} \mathrm{Hg}$. The electrocardiogram, chest roentgenogram and routine laboratory tests were normal. Additional laboratory tests for hypercoagulability and autoimmune diseases were also all normal.

Nine hours after the onset of symptoms, an MRI showed a small lesion affecting the left posterior area of the cervical spinal cord at the C1 level (fig. 1). On DWI, the lesion appeared as a punctiform hypersignal with a decreased apparent diffusion coefficient. In the FLAIR sequence, the lesion appeared as a hypersignal. The lesion did not enhance after contrast administration on $\mathrm{T}_{1}$-weighted images. MR angiography showed a hypoplastic left vertebral artery, terminating in the posterior inferior cerebellar artery (PICA) with no connection to the basilar artery. The cervical Doppler and cervical axial $\mathrm{T}_{1}$-weighted sequences were normal, as were the heart and aortic arch on the transoesophageal echocardiogram. Standard cerebrospinal fluid analysis was normal and no intrathecal immunoglobulin synthesis was found. A small upper cervical infarction in the left posterior spinal artery territory was diagnosed.

During hospitalisation, sensory symptoms gradually improved, but they still persisted at discharge. Aspirin, statin and anti-hypertension treatments were started in the acute phase and continued after discharge. During follow-up, sensory soft symptoms persisted, and an MRI at 3 months showed no change in the evolution of the lesion or the appearance of the left vertebral artery.

\section{Discussion}

Our case confirms that posterior spinal artery syndrome is difficult to identify clinically [3]. Indeed, even if sensory patterns appear as cardinal signs, our case emphasises that their distribution can be very variable. Unilateral spinal cord infarctions with hemisensory symptoms, as in our case, are rarely reported, and sensitive symptoms are more frequently associated with weakness of the limbs [2, 4, 5]. In most cases, spinal cord infarcts induce bilateral sensory disturbance because lesions are more frequently bilateral or because the spinothalamic tract and dorsal column are both affected by a unilateral lesion [5]. In our case, the first symptoms consisted of acute paraesthesia located in the left occipital and temporal area of the head and the left side of the nape of the neck. To our knowledge, this kind of clinical onset has not yet been reported in cases of posterior spinal infarction.

Furthermore, the isolated sensory symptoms extended, and the clinical picture was similar to that encountered in pure sensory stroke (PSS) with faciobrachial distribution [6]. PSS is a well-defined clinical syndrome in which hemisensory symptoms predominate without the appearance of other major neurological signs [7]. In most cases, PSS is a consequence of thalamic stroke, but the brain stem and cerebral cortex have been widely reported as other potential sites [8, 9]. Nevertheless, in our patient, although the initial onset of symptoms was abrupt, secondary sensory symptoms developed gradually within a few hours. This initial evolution, which is rare in cerebral infarction, seems to be more common in the case of medullary infarction $[4,10]$.

As shown on sagittal $\mathrm{T}_{2}$-weighted images, the lesion was strictly limited to the $\mathrm{C} 1$ level, whereas in most cases, the infarct affected several levels of the spinal cord or a 
combination of the medulla and upper cervical cord $[1,3,11,12]$. There is a good correlation between the sensory symptoms, their systematisation and the anatomical location of the infarct. In terms of anatomical structures, the lesion affected the left posterior horn of the grey matter, a lateral part of the left posterior column, defined as the cuneate fasciculus, and a restricted posterior part of the left lateral column. At this level, the left nucleus of $\mathrm{V}$ was damaged. As previously reported, these are the most frequently affected structures in cases of spinal posterior infarction $[3,5]$. The left anterior horn, spinothalamic tract and corticospinal tract were unaffected.

Paraesthesia in the C2 dermatome, as observed in Arnold's neuralgia, was due to the posterior horn involvement, defined as the lesional syndrome of spinal involvement. Paraesthesia in the trunk and arm can be assimilated as the sublesional syndrome due to the damage to the lateral part of the dorsal column. The systematisation of symptoms in our patient confirms the hypotheses that have already been reported on the somatotopy of the dorsal column, in which fibres from the rostral parts of the body are located laterally $[13,14]$. Our patient did not have any objective epicritic sensory disturbances, confirming that the dorsal column cannot be the only ascending pathway required to transmit epicritic information [13]. The symptoms appearing on the left side of the face were due to the damage to the descending tract and nucleus of $\mathrm{V}$, which are present at this level of the spinal cord. These symptoms are defined as the supralesional syndrome of this spinal infarct.

Most posterior spinal artery infarcts are the consequence of an aortic or vertebral artery disease [5]. Intrinsic diseases of the intraspinal arteries or a cardioembolism are much less common $[5,15]$. After an extensive diagnostic workup, we did not find any evidence of artery dissection, a cardioembolic source or inflammatory arteritis, confirming that the cause of this type of infarct remains unknown [15]. Nevertheless, because the left vertebral artery was smaller than the right one, a diagnosis of dissection was initially put forward. Despite having had a motorcycle accident a few days earlier, the patient had no cervical pain or headaches, and no parietal haematoma was found on the MRA or axial $\mathrm{T}_{1}$-weighted images of the neck. Moreover, the appearance of the left vertebral artery was unchanged in an MRA test 3 months later.

The left vertebral artery terminated in the PICA. This particular arterial disposition raises questions as to the territory supplied by the vertebral artery and the origin of the posterior spinal artery. According to Lasjaunias et al. [16], some hypoplastic vertebral arteries supply only the cervical territories, such as the upper cervical cord. In these cases, no branches are involved in the supply of the medulla oblongata. These data could explain why, in our patient, the infarct did not extend to the medulla oblongata. Usually, posterior spinal arteries arise either from the vertebral artery or from the PICA $[16,17]$. To our knowledge, no more specific anatomical information is available regarding the origin of posterior spinal arteries in cases of vertebral arteries terminating in the PICA.

In conclusion, our case illustrates an unusual clinical picture of posterior spinal artery infarction and confirms the difficulty of establishing this diagnosis. This case highlights the complexity of the posterior spinal artery territory, especially at the level of the upper cervical cord. 

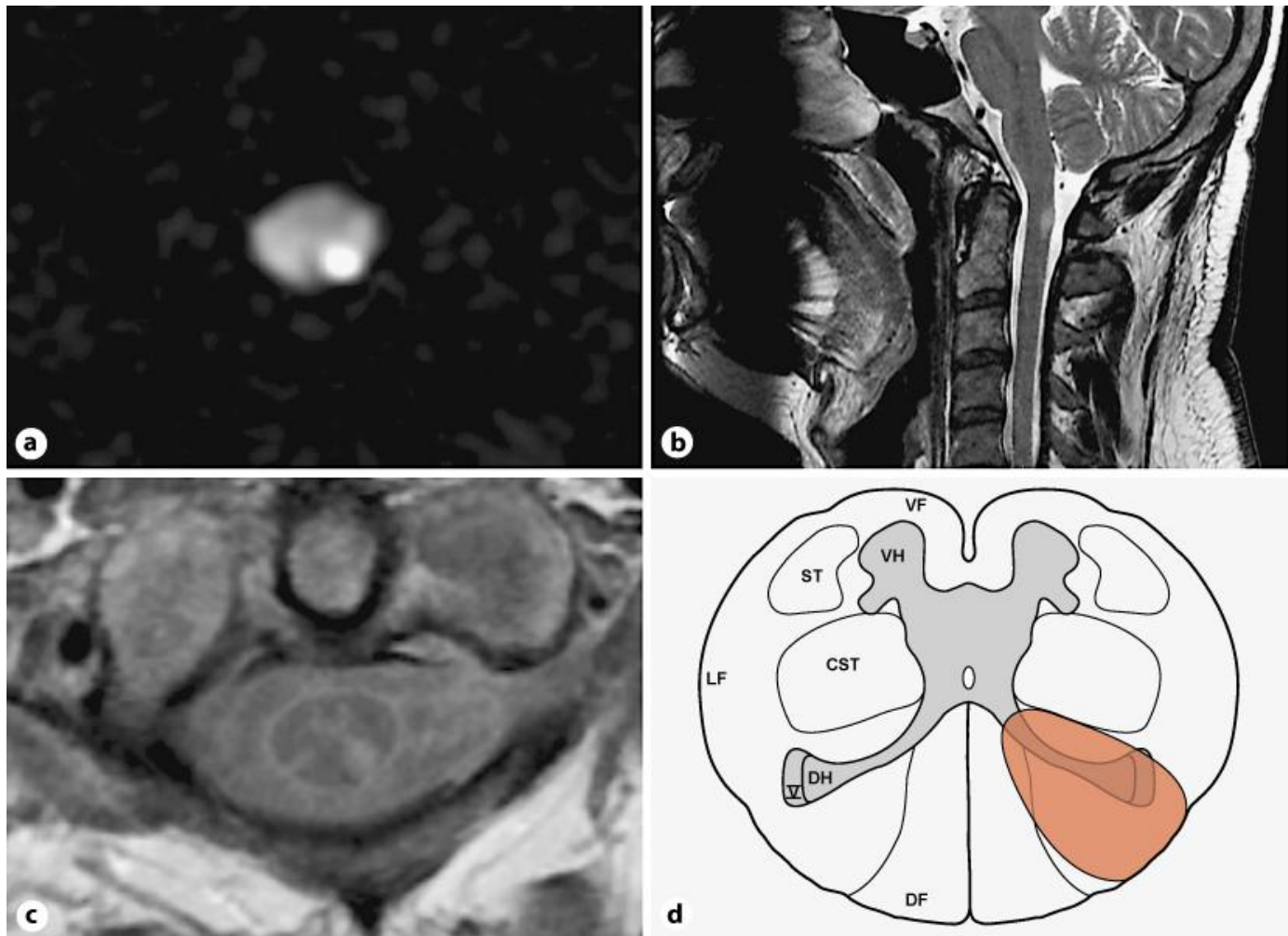

Fig. 1. a Axial DWI showing an infarct in the left posterior part of the spinal cord. $\mathbf{b}$ Sagittal $\mathrm{T}_{2}$ weighted image showing that the lesion was strictly limited to the C1 level. c Axial FLAIR image showing that the lesion involved the left posterior horn of the grey matter, a lateral part of the posterior column and a restricted posterior part of the lateral column. $\mathbf{d}$ Drawing of an axial section of the spinal cord at the $\mathrm{C} 1$ level. The infarct lesion is drawn in red. VF = Ventral funiculus; $\mathrm{LF}=$ lateral funiculus; $\mathrm{DF}=$ dorsal funiculus; $\mathrm{VH}=$ ventral horn of the grey matter; $\mathrm{DH}=$ dorsal horn of the grey matter; ST = spinothalamic tract; $\mathrm{CST}=$ corticospinal tract; $\mathrm{V}=$ nucleus of the trigeminal nerve.

\section{References}

1 Weidauer S, Nichtweiss M, Lanfermann H, Zanella FE: Spinal cord infarction: MR imaging and clinical features in 16 cases. Neuroradiology 2002;44:851-857.

-2 Kaneki M, Inoue K, Shimizu T, Mannen T: Infarction of the unilateral posterior horn and lateral column of the spinal cord with sparing of posterior columns: demonstration by MRI. J Neurol Neurosurg Psychiatry 1994;57:629-631.

3 Gutowski NJ, Murphy RP, Beale DJ: Unilateral upper cervical posterior spinal artery syndrome following sneezing. J Neurol Neurosurg Psychiatry 1992;55:841-843.

-4 Masson C, Pruvo JP, Meder JF, Cordonnier C, Touzé E, De la Sayette V, Giroud M, Mas JL, Leys D; Study Group on Spinal Cord Infarction of the French Neurovascular Society: Spinal cord infarction: clinical and magnetic resonance imaging findings and short term outcome. J Neurol Neurosurg Psychiatry 2004;75:1431-1435.

5 Leys D, Weerts JG, Pruvo JP: Spinal ischemic strokes; in Ginsberg MD, Bogousslavsky J (eds): Cerebrovascular Disease: Pathophysiology, Diagnosis and Management. Cambridge, Blackwell Scientific Publications, Inc., 1998, pp 1560-1568.

6 Kim JS: Pure sensory stroke. Clinical -radiological correlates of 21 cases. Stroke 1992;23:983-987.

7 Fisher CM: Pure sensory stroke involving face, arm and leg. Neurology 1965;15:76-80. 
8 Hommel M, Besson G, Pollak P, Borgel F, Le Bas JF, Perret J: Pure sensory stroke due to a pontine lacune. Stroke 1989;20:406-408.

9 Bassetti C, Bogousslavsky J, Regli F: Sensory syndromes in parietal stroke. Neurology 1993;43:1942 1949.

10 Novy J, Carruzzo A, Maeder P, Bogousslavsky J: Spinal cord ischemia : clinical and imaging patterns, pathogenesis, and outcomes in 27 patients. Arch Neurol 2006;63:1113-1120.

11 Pryse-Phillips W: Infarction of the medulla and cervical cord after fitness exercises. Stroke 1989;20:292294.

12 Mandrioli J, Zini A, Cavalleri F, Nichelli P, Panzetti P: Bilateral posterior medullary and cervical stroke: a case report. Neurol Sci 2006;27:281-283.

13 Davidoff RA: The dorsal columns. Neurology 1989;39:1377-1385.

14 Smith MC, Deacon P: Topographical anatomy of the posterior columns of the spinal cord in man. Brain 1984;107:671-698.

15 Cheshire WP, Santos CC, Massey EW, Howard JF Jr: Spinal cord infarction: etiology and outcome. Neurology 1996;47:321-330.

16 Lasjaunias P, Berenstein A, ter Brugge KG: Spinal and spinal cord arteries and veins; in Lasjaunias P, Berenstein A, ter Brugge KG (eds): Surgical Neuroangiography: Clinical Vascular Anatomy and Variations, ed 2. Berlin, Springer, vol 1, 2001, pp 73-164.

17 Tatu L, Vuillier F, Moulin T: Chapter 13. Anatomy of the circulation of the brain and spinal cord. Handb Clin Neurol 2009;92:247-281. 